Décadrages Décadrages

cinéma, à travers champs Cinéma, à travers champs

4-5 | 2005

David Lynch

\title{
Du roman à l'écran : une question de droit
} d'auteur...

\section{Luc Amgwerd}

\section{OpenEdition}

1 Journals

Édition électronique

URL : https://journals.openedition.org/decadrages/617

DOI : $10.4000 /$ decadrages. 617

ISSN : 2297-5977

Éditeur

Association Décadrages

Édition imprimée

Date de publication : 10 avril 2005

Pagination : 147-153

ISBN : 978-2-9700582-1-2

ISSN : 2235-7823

Référence électronique

Luc Amgwerd, «Du roman à l'écran : une question de droit d'auteur... », Décadrages [En ligne], 4-5 |

2005, mis en ligne le 21 janvier 2013, consulté le 27 mars 2022. URL : http://journals.openedition.org/ decadrages/617; DOI : https://doi.org/10.4000/decadrages.617 


\section{Du roman à l'écran: une question de droit}

\section{d'auteur...}

\begin{abstract}
"Plus les qualités littéraires de l'œuvre sont importantes et décisives, plus l'adaptation en bouleverse l'équilibre, plus aussi elle exige de talent créateur pour reconstruire selon un équilibre nouveau, non point identique, mais équivalent à l'ancien»1.
\end{abstract}

La littérature n'a cessé de fournir au cinéma un réservoir sans fond de fictions et de personnages: Robinson Crusoé, Blow $U_{p}$, Le Vicomte de Valmont, La Chambre verte, Crash!, Tyler Durden, Les marins perdus... Belles béquilles littéraires! Cependant, imaginer qu'un roman ${ }^{2}$ est une histoire toute prête pour le cinéma est une illusion. Illusion pour les scénaristes, metteurs en scène et réalisateurs, mais également pour les juristes... Pour ces derniers, il ne s'agit pas seulement de se demander combien valent les droits, mais également de s'interroger sur les pratiques et les astuces en la matière...

\section{L'adaptation: un régime de semi-liberté !}

«La technologie implique que vous pouvez faire certaines choses facilement, mais vous ne pouvez pas facilement les faire licitement» 3 . Se poser la question de la protection des œuvres, littéraires, visuelles ou artistiques, implique inévitablement de se pencher sur les législations du droit d'auteur ${ }^{4}$. Plonger rapidement dans les réflexions initiales du droit d'auteur permet d'en comprendre les enjeux actuels... et le lieu de tension qu'il n'a cessé d'être!

Les premières réflexions sur le droit d'auteur, tel qu'on le connait de nos jours, remontent au milieu du XIXe siècle. Ecrivains, éditeurs, philosophes, économistes, juristes se mobilisent et alimentent les premiers débats sur la protection des créations culturelles. Comment diffuser les œuvres et offrir simultanément aux artistes une contrepartie à leur travail ? Les débats se focalisent sur la notion de propriété. Deux
1 André Bazin, "Pour un cinéma impur " [1952], in Qu'est-ce que le cinéma?, Editions du Cerf, Paris, 1994, p. 97.

2 Nous nous proposons de regrouper sous le terme générique de "roman" toute œuvre à caractère "littéraire" (au sens large): nouvelles, bandes dessinées, biographies, pièces de théâtre, journal intime, essais, etc. Ce regroupement facilitera l'exposé, et semble en outre être en phase avec la grande majorité des adaptations littéraires actuelles.

3 "Technology means you can now do things easily; but you couldn't easily do them legally", Lawrence Lessig, Free Culture, Penguin Press, New York, 2004, p. 105 (ou sur http://www. free-culture.cc/).

4 La Convention de Berne (1896), http:// www.wipo.int/treaties/en/index.html, est le cadre juridique international; la Directive sur l'harmonisation de certains aspects du droit d'auteur et des droits voisins dans la société de l'information (2001) concerne l'Europe, et la loi sur le droit d'auteur et les droits voisins (1992) la Suisse (RS. 232.1, http://www.admin.ch/ ch/f/rs/c231_1.html). 
$\mathbf{5}$ Les mots choisis par Le Chapellier lors de son discours à l'Assemblée nationale, lors des débats sur la loi relative aux spectacles en sont fort révélateurs: "la plus sacrée, la plus inattaquable, la plus personnelle de toutes les propriétés, est l'ouvrage, le fruit de la pensée de l'homme".

6 Autant la question de la durée du droit d'auteur (voir le personnage Mickey Mouse, né en 1928 aux Etats-Unis qui a "poussé" le Congrès américain à prolonger la durée de protection des droits, par la création du Sonny Bono Copyright Term Extension) que celle de la dualité droits patrimoniaux / droits moraux (par exemple, la Convention internationale la plus ambitieuse en la matière, les Accords ADPIC, http://www.wto.org/french/tratop_f/trips_f/ trips_f.htm, ne traite que de l'aspect patrimonial...) alimentent les débats actuels sur le droit d'auteur.

7 La loi sur le droit d'auteur (art. 29, al. 2 lit. a) prévoit un régime de cinquante ans pour les logiciels.

8 Jean Cocteau, Le coq et l'Arlequin: notes autour de la musique, Stock, Paris, 1979 [1918].

9 En Suisse, la loi sur le droit d'auteur (LDA), du 9 octobre 1992, http://www.admin.ch/ch/f/rs/ c231_1.html, fait foi. conceptions s'affrontent. La première est inspirée de la philosophie de John Locke: la propriété est perçue comme un droit total, sacré et immuable ${ }^{5}$. La seconde, inscrite dans la lignée des pensées de Pascal et Montesquieu, est davantage tournée vers l'utilité sociale. La notion de "propriété» est alors modulée par des accords entre parties.

Cette controverse n'est pas que théorique... pas plus qu'historique 6 ! Elle "cache» deux questions décisives, à l'orée des premières législations sur le droit d'auteur. La première concerne la manière de concilier les intérêts personnels de l'auteur (le respect de l'œuvre ou celui de la paternité de l'auteur) et la propriété en tant que telle. Pour cela, les premiers législateurs ont imaginé un droit d'auteur construit à l'image d'un "arbuste à deux branches»: on trouve d'une part les prérogatives personnelles de l'auteur (droit moral), d'autre part les aspects plus utilitaires qui interviennent dans l'échange (droit patrimonial).

Reste alors la deuxième question, celle de la durée des droits. Les droits d'auteur sont-ils perpétuels? La réponse est évidemment non. Une durée limitée est légitimée par le fait que les œuvres ont vocation d'éducation et de culture et appartiennent au domaine public après un certain laps de temps. En Suisse, les œuvres sont protégées septante ans après le décès de l'auteur, à l'exception des logiciels 7 .

Paradoxe ou subtil mélange? Le droit d'auteur est un jeu d'équilibre perpétuel et unique: il attribue une certaine forme de propriété à l'auteur de l'œuvre et permet simultanément au public de "profiter de l'imaginaire» des artistes. Il apparaît ainsi clairement que le système choisit de concilier à la fois les intérêts des auteurs afin qu'ils puissent tirer avantage de leurs travaux, tant au niveau de la notoriété qu'au niveau pécuniaire, et ceux du public en permettant à celui-ci un accès aux créations protégées.

\section{La création est protégée}

«Un artiste original ne doit pas copier. Il n'a donc qu'à copier pour être original» 8 .

Les juristes ne sont pas les plus habiles pour élaguer les définitions et les principes de base... On tentera donc de ne pas trop s'attarder sur les contours légaux!

Les législations sur le droit d'auteur ${ }^{9}$ postulent que le travail des créateurs est protégé et réglementé. Toutes les lois en la matière ont tout d'abord pris soin de définir ce qu'est une œuvre. Est donc considérée comme une ouvre au sens de la loi, une création de l'esprit, littéraire ou artistique et qui est originale. A ces trois éléments fondamentaux, on peut ajouter que ces créations doivent être perceptibles. A contrario, les idées 
ne peuvent pas être protégées; les juristes ont coutume de dire qu'elles sont laissées à "libre parcours»10. La Cantatrice chauve de Ionesco, la Villa Savoye du Corbusier, le thème de Mannix par Lalo Schiffrin et les décors de H.R. Giger... ces quelques exemples sont des créations protégées par le droit d'auteur!

Un roman et un film entrent naturellement dans le champ de protection du droit d'auteur. Une œuvre cinématographique mérite quelques précisions, car, dans ce cas, la protection est complexe. D’un point de vue théorique, réalisateurs, metteurs en scène, décorateurs, costumiers, monteurs, etc. sont tous coauteurs de l'œuvre. Des réserves sont généralement admises, à la fois pour les scénaristes et les auteurs de la musique. Soit. Mais comment cela se passe-t-il concrètement? Le décorateur peut-il revendiquer des droits sur son seul travail ? En résumé et de manière générale, les droits sont cédés au producteur, mais chaque artiste conserve une propriété sur sa contribution, à condition de ne pas porter préjudice à la création dans son ensemble... On l'imagine bien, les contrats et leur mise en œuvre est un véritable régal pour les services juridiques attachés aux productions!

\section{Adapter, c'est créer? Oui.}

La loi a pensé pour la notion "traditionnelle» de l'œuvre une "petite sœur». Il s'agit de l'œuvre dérivée (les termes d'adaptation, d'œuvre de seconde main et d'œuvre composite en sont des synonymes). L'œuvre dérivée est une œuvre à part entière: elle est une création de l'esprit, originale, dotée d'un caractère littéraire ou artistique, mais conçue à partir d'une œuvre préexistante et reconnaissable. Ainsi, dès le moment où l'œuvre reprise est reconnaissable, il est nécessaire d'obtenir l'autorisation de son auteur. A la seule lecture du texte de loi, on se doute bien que le terme "reconnaissable» fascine (ou crispe!) autant les artistes que les juristes, et que la réalité est plus capricieuse que l'énoncé!

On l'imagine bien: l'œuvre dérivée, ou adaptation, est un grand écart. Grand écart entre le simple fait de reprendre une œuvre pour la proposer au public par exemple (la loi suisse parle d'utilisation, au sens de l'art. 10 LDA) et le besoin pour le créateur d'y apporter sa touche personnelle! Autrement dit, dans un travail d'adaptation, ce sont «deux originalités qui cohabitent»: celle de l'œuvre initiale et celle de la nouvelle. C’est pourquoi la parodie est considérée comme une œuvre dérivée, car elle reprend une création connue et la dote d'un effet humoristique. La bande dessinée de Tardi, Voyage au bout de la nuit, est également une œuvre dérivée, car bien que se basant sur le roman de Céline, elle apporte des modifications et l'aménagement est original. Mais jusqu'où
10 L'expression de "libre parcours" signifie que les idées ne peuvent être "cadenassées" par le droit d'auteur. II est de l'intérêt de la démocratie, de l'art et du progrès que les idées puissent circuler librement. Par contre, la "mise en forme ou en musique" des idées constitue pour les juristes l'acte de création. C'est à ce moment-là que l'on peut considérer que l'œuvre est perceptible. 
11 Claude Chabrol, François Guérif, Comment faire un film, Editions Payot \& Rivages, Paris, 2003, p. 22.

12 La loi suisse sur le droit d'auteur ne consacre aucune disposition particulière à ce principe, au contraire du droit allemand par exemple (art. 24), mais autant le Tribunal fédéral que les auteurs de doctrine sont unanimes à ce sujet. peuvent aller les modifications? Les critères quantitatifs (50 secondes, 5 paragraphes, 10 ressemblances flagrantes, etc.) permettent-ils de poser des barrières? Non, le droit n'aime pas les solutions toutes faites... Il lui préfère le cas par cas et une estimation qualitative. Pour ce faire, la loi offre à l'auteur initial qui se sent lésé dans son droit une disposition de poids : le devoir de fidélité que doit respecter tout utilisateur (la loi suisse parle de devoir d'intégrité, art. 11 LDA). Claude Chabrol donne sur ce point le conseil suivant:

«En général, la clé est d'être extrêmement fidèle et de se poser les questions uniquement par rapport au bouquin que l'on adapte» $\mathbf{1 1}$.

\section{S'inspirer, c'est adapter? Non.}

Reconnaissable, disions-nous. C'est donc le critère que la loi a choisi pour différencier une adaptation d'une œuvre "qui s'inspire de». La loi n’a jamais cherché à "bris(d ?)er» la création et la circulation des idées, et a donc reconnu, bien qu'entre les lignes ${ }^{\mathbf{1 2}}$, le principe de la libre utilisation.

Ainsi, dans le cas où une ou plusieurs œuvres préexistantes ne représentent que le point de départ vers une nouvelle création, celle-ci est une œuvre à part entière. On ne parle plus d'adaptation, de fidélité et d'autorisation... Ce ne sont plus deux œuvres qui cohabitent, mais bien une qui se distancie par rapport à l'autre et la nouvelle œuvre est protégée comme telle. Prospero's Books de Peter Greenaway (1991) est-il une illustration de la libre utilisation? Pas si sûr, car bien que différente de la pièce de William Shakespeare, La tempête, le film n'en reste pas moins fidèle dans l'esprit... Par contre, il est certain que l'œuvre de Shakespeare appartient au domaine public et que l'autorisation pour une adaptation n'est pas nécessaire!

\section{Citer et couper/coller, c'est adapter? Non.}

«La règle du jeu fait ici partie de la culture» $\mathbf{1 3}$.

13 André Lucas évoquant les questions de l'interdit et du droit d'auteur, in Critique, Copier, voler: les plagiaires, $\mathrm{n}^{\circ}$ 663-664, août-septembre 2002, p. 601.
Phénomènes littéraires ou détournements anecdotiques, que dire des collages, échantillonnages et emprunts mineurs? L'art n'a cessé de panacher, à croire que les auteurs aiment re-créer autour de bribes épinglées ça et là. Ayons une interprétation large. Sorte de clin d'œil qui favorise l'émancipation culturelle, "une citation est une lecture", disait Godard. Dans ce sens, elle est autorisée et le droit d'auteur l'a consacrée au rang d'exception (art. $25 \mathrm{LDA})$. Cependant, comme dans tout régime d'exception, l'interprétation stricte de la disposition est de rigueur: un extrait n'est pas une citation et l'auteur se doit de respecter l'intégrité de l'emprunt et citer la source. Qu'en est-il des collages littéraires d'Aragon, 
des détournements situationnistes, du «découper-coller» de Godard et des pratiques de sound\&picture-sampling de Coldcut? Deux "plages de liberté juridique» se dessinent pour les créateurs: d'une part, ils bénéficient de la liberté de citer, certes «bien encadrée», et d'autre part, de la liberté d'utilisation. Dans cette dernière situation, l'emprunt ne devient qu'une source d'inspiration et se fait «oublier» dans la création nouvelle! La frontière est souvent ténue entre l'emprunt autorisé et non-autorisé...

\section{L'interdit: le plagiat}

"Il n'est ni besoin de juge, ni de défenseur pour constater ton plagiat. Ton livre se dresse contre toi et crie que tu es un voleur».

Ainsi s'adressa Martial à Fidentius (Epigrammes, I, 53) pour dénoncer les premiers plagiats. Le terme a pris de l'âge, mais les pratiques restent constantes: c'est bel et bien l'insuffisance, le vide qui dévisage le plagiaire! Là encore le droit peine à se placer en terrain "scientifique» et s'arme du droit moral, via le devoir d'intégrité, pour juger de la copie. Au cas par cas, en comparant les similarités importantes de l'original avec la copie, en se retranchant donc vers une estimation qualitative, le juge est le dernier recours.

«Les optimistes parleront d'aléa judiciaire, les pessimistes de loterie...»14.

\section{Le contrat d'adaptation cinématographique : un parcours du combattant !}

Comment procéder donc si l'on désire adapter un roman à l'écran? Faut-il s'inspirer des pratiques de Fritz Lang, David Cronenberg, JeanLuc Godard ou des expériences panachées de Paul Auster15 ? Mis à part les jalons exposés plus haut et les longues clauses contractuelles, il n'y a peut-être qu'une seule règle dans ce subtil exercice: il faut aimer passionnément le roman... et savoir pourquoi il en est ainsi. De manière plus pragmatique, il est évident qu'il y a des styles ou des auteurs dont les récits sont plus aisément "adaptables» à l'écran: Stephen King et Philippe K. Dick caracolent en tête, Victor Hugo, Alexandre Dumas, et même Georges Simenon ont également une fière filmographie. Au-delà du récit, comment recréer une musicalité proche du roman? Ce ne sont pas les juristes qui vont nous aiguiller sur le sujet... Par contre, trois "scénarios juridiques» permettront de se rendre compte que le numéro d'équilibriste nécessité par l'adaptation littéraire se double généralement d'un parcours du combattant!

«Le talent, certes, n'est pas fonction de la fidélité, mais je ne conçois d'adaptation valable qu'écrite par un homme de cinéma» $\mathbf{1 6}$.
14 André Lucas (2002), ibid., p. 597.

15 Au-delà de ses fameux romans, Paul Auster a, entre autres, réalisé Lulu in the Bridge (2001) et scénarise actuellement Monster of Longwood (Partice Chéreau, 2004).

16 François Truffaut, "Une certaine tendance du cinéma français", in Cahiers du cinéma, n³1, janvier 1954. 
17 A contrario, les romans de plus de septante ans sont hors du champ du droit d'auteur et appartiennent donc au domaine public, au regard du droit suisse.

18 Suissimage fournit un contrat-type et un commentaire sur son site, http://www. suissimage.ch/.
Une adaptation cinématographique ou audiovisuelle met en relation le couple auteur/éditeur - ce dernier est généralement titulaire des droits, sur la base du contrat d'édition - et le producteur. Les contrats ficelant ce rapport varient naturellement de fois en fois, de pays en pays, mais quelques grandes lignes peuvent être présentées. Un contrat d'adaptation cinématographique ou audiovisuelle permet donc à la production de «transcrire» un roman, œuvre protégée $\mathbf{1 7}$, à l'écran. Les négociations se révèlent "âpres", car c'est dans ce document $\mathbf{1 8}$ que se définissent toutes les modalités: exclusivité de l'adaptation, prix des droits, produits dérivés, participation de l'auteur au scénario... La signature du contrat permet notamment à la production de commander l'écriture du scénario et de rechercher un distributeur. Mais la question est sur toutes les lèvres: combien coûtent des droits d'adaptation? Joker! Suissimage, dans le commentaire du contrat-type, avance qu'il est généralement question d'un forfait et que celui-ci équivaut entre $2 \%$ et $5 \%$ du budget du film (la participation aux recettes est exclue dans ce calcul). Cependant, une chose est sûre: le prix dépend davantage de la notoriété de l'auteur que de l'intrigue!

\section{L'esprit de l'œuvre absent: que faire?}

En suivant le scénario précédent, imaginons que l'auteur initial soit «irrité» de l'utilisation qui est faite de son œuvre ou de parties de celleci, malgré le contrat signé. Par exemple, l'intrigue et les personnages sont calqués sur le roman, mais la cadence ou les ambiances sont très lointaines... Que peut faire l'auteur? La logique commerciale et la réalité juridique peuvent s'avérer coriaces : le contrat d'adaptation est quasi toujours exclusif. Cela signifie que l'auteur ne peut se tourner vers une autre production pour une nouvelle adaptation. De plus, invoquer une violation de l'intégrité de l'œuvre est une (dé)marche longue et incertaine dans les allées de la justice... A cela s'ajoute le fait que les clauses contractuelles sont souvent alambiquées pour les non-juristes. Bonjour patience! Par cet exemple, on se rend bien compte que la négociation du contrat n'est pas un élément à prendre à la légère...

\section{Adapter l'inadaptable!}

Les Duellistes (The Duellists, Ridley Scott, 1977), tiré de la nouvelle de Joseph Conrad (Le duel); Blow Up de Michelangelo Antonioni (1966), réalisé sur la base des quelques pages des Fils de la Vierge de Julio Cortazar; Le festin nu (Naked Lunch) de William S. Burroughs "repris» par David Cronenberg dans le film homonyme (1991): ou comment adapter/adopter "l'inadaptable». Dans cet exercice de style, il peut 
sembler inapproprié de parler de fidélité et de trahison. La densité littéraire du récit guide "difficilement» le scénario, car la liberté donnée par le récit est plus importante. "L'histoire adaptée devient autre»19, commente Tonino Benacquista, auteur notamment des Morsures de l'Aube (2001). Oui, dans ce cas, on flirte avec la mention "inspirée de" ou «librement adapté de», là où scénariste et réalisateur tentent de restituer la profondeur secrète d'un récit. Le droit d'auteur pourrait ne pas s'en mêler, mais l'honnêteté intellectuelle et la sécurité juridique commandent néanmoins l'achat des droits.

"La profession de Noiret, le sens général de l'intrigue et l'équivalent du casse-autos. C'est peu pour justifier l'achat des droits» 20.

\section{Cinéma vs littérature?}

"〈J'ai dix-huit jours, je me souviens... , ça ne se dessine pas. Le texte est devenu la colonne vertébrale de l'album. Ensuite, tout naturellement, j’ai raisonné en termes de plans» $\mathbf{2 1}$.

Cinéma et littérature ont toujours entretenu un rapport complexe et complice. "Hitchkock vaut-il Chateaubriand?" se demandait Godard dans les colonnes du Nouvel Observateur ${ }^{22}$, Michel Houellebecq a-t-il changé d'éditeur pour s'assurer l'adaptation à l'écran - déjà signée avec une maison-sœur de l'éditeur - de son prochain roman? "Pourquoi être cinéaste, si l'on peut être romancier?» $\mathbf{2 3}$ s'interroge Eric Rohmer, ou, à l'inverse, comment s'identifier à Judy Davis dans Barton Fink (Joel Cohen, 1991)? La question ne devrait pas seulement se poser en termes de rivalité. Un roman qui «fait des petits» au cinéma peut révéler une famille harmonieuse. Adaptation stricte ou adaptation lointaine. Les films offrent bien souvent une seconde vie, méritée ou bancale, à des récits littéraires. Mais au-delà des phrasés juridiques et des ventes qui rebondissent, souvenons-nous que le travail d'adaptation cinématographique utilise bien plus que la seule plume pour outil, que la création de ce nouvel univers est une délicieuse acrobatie... et est naturellement protégeable!
19 Citation reprise du dossier spécial que Synopsis ( $n^{\circ} 25$, mai 2003, p. 38) a consacré à l'adaptation littéraire.

20 Claude Chabrol, évoquant son expérience dans Masques (1987), adapté de L'insoupçonnable Grandison de Charlotte Armstrong, in Claude Chabrol, François Guérif, Comment faire un film, op. cit., p. 22.

21 Enki Bilal, s'exprimant sur l'apport du cinéma à son travail, cf. www.humano.com/bilal.

22 Cf. http://artsetspectacles.nouvelobs.com/ dossier/dossier2089_092.html.

23 Eric Rohmer, Six contes moraux, Editions de I'Herne, Paris, 1974, p. 14. 